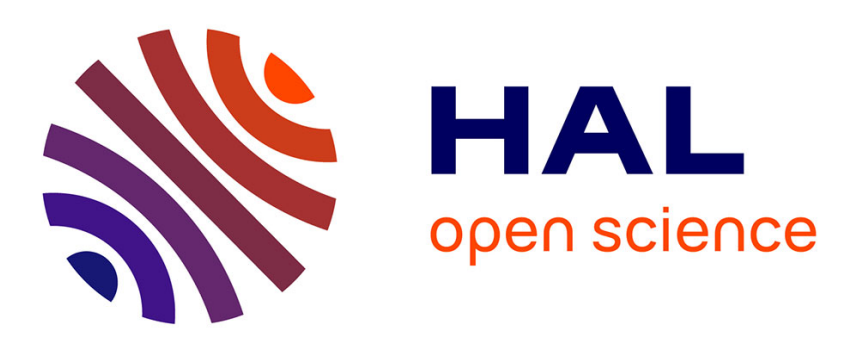

\title{
Une architecture de système d'information collaboratif pour la gestion de crise. Approche basée sur la médiation des systèmes
}

Sébastien Truptil, Frederick Benaben, Hervé Pingaud

\section{To cite this version:}

Sébastien Truptil, Frederick Benaben, Hervé Pingaud. Une architecture de système d'information collaboratif pour la gestion de crise. Approche basée sur la médiation des systèmes. Ingéniérie des Systèmes d'Information, 2010, 15 (3), pp.11 - 36. 10.3166/isi.15.3.11-36 . hal-01758214

\author{
HAL Id: hal-01758214 \\ https://hal.science/hal-01758214
}

Submitted on 7 Nov 2019

HAL is a multi-disciplinary open access archive for the deposit and dissemination of scientific research documents, whether they are published or not. The documents may come from teaching and research institutions in France or abroad, or from public or private research centers.
L'archive ouverte pluridisciplinaire HAL, est destinée au dépôt et à la diffusion de documents scientifiques de niveau recherche, publiés ou non, émanant des établissements d'enseignement et de recherche français ou étrangers, des laboratoires publics ou privés. 


\title{
Une architecture de système d'information collaboratif pour la gestion de crise
}

\author{
Approche basée sur la médiation des systèmes
}

\author{
Sébastien Truptil — Frédérick Bénaben — Hervé Pingaud \\ Centre de Génie Industriel, \\ Université de Toulouse, Ecole des Mines d'Albi-Carmaux, \\ Campus Jarlard - 81013 Albi \\ \{truptil,Bénaben,pingaud\}@enstimac.fr
}

\begin{abstract}
RÉSUMÉ. Un objectif du projet de recherches ISyCri (ANR-CSOSG) est de définir une architecture de système d'information collaboratif afin d'aider à la résolution d'une crise dans laquelle plusieurs partenaires sont impliqués. Après avoir décrit certaines spécificités liées au contexte, notre recherche étudie le problème au moyen de deux concepts structurants: un système de systèmes permettant de travailler à partir de systèmes contributeurs relativement autonomes, induisant une exigence sur des capacités d'interopérabilité dans l'espace de formulation du problème; un système de médiation tirant profit de cette interopérabilité et dont nous montrons que la conception peut être renouvelée facilitant ainsi le développement de solutions agiles. En effet, comme une crise est un phénomène évolutif, l'agilité du système est déterminée par la possibilité de configurer le médiateur en ligne permettant de respecter des exigences nouvelles au fil de l'eau. A cette fin, une approche basée sur l'ingénierie dirigée par les modèles est mise en ouvre. Le médiateur coordonne continuellement l'exécution des processus collaboratifs voulus par les acteurs : il participe à l'invocation des services qu'ils exposent ainsi qu'à l'échange et l'interprétation des informations au cœur même du dispositif de gestion de crise.

ABSTRACT. One objective of the ISyCri project is to design an information system for several partners who have to solve, or at least to reduce, a crisis into which they are involved. This system must not only support communication between actors, but also coordinate their activities. It might be considered like a system of systems for which interoperability between partners is a key factor. As a main component of such a critical system, a Mediation Information System (MIS) is defined at the core of the ISyCri architecture, with the aim to coordinate actors' services and information exchange. Because a crisis is intrinsically an evolutionary phenomenon, the system shall remain compliant with the expectations of the actors. We propose to make an adaptation of the MIS on the fly, using a MDE approach for platform configuration. By this way, the MIS has some capacity to adapt itself to changes on system requirements.
\end{abstract}

MOTS-CLÉS : système d'information, médiation, gestion de crise.

KEYWORDS: information system, crisis management, mediation. 


\section{Définition du sujet}

\subsection{Contexte de l'étude}

Une crise peut revêtir différentes natures : militaire, politique, économique, sanitaire, sociale, technique ou humanitaire (Devlin, 2006). Cependant, quelle que soit sa nature, elle est le résultat d'une instabilité non tolérable de l'écosystème impacté. La crise résulte alors de l'occurrence d'un événement déclencheur au sein d'un environnement présentant une certaine vulnérabilité (Tomasini et al, 2004 ; Lagadec, 1992).

Les événements déclencheurs, appelés selon les contextes et les sources « calamités » (Ryfman, 1999), « incidents » (Devlin, 2006) ou «accidents » (Lagadec, 1992), peuvent être classifiés en deux grandes catégories (Tomasini et al, 2006) : (i) celles dont la survenue est brutale (ouragans, éruptions volcaniques, catastrophes industriels, tremblements de terre...) (ii) celles qui émergent progressivement (sécheresses, épidémies, famines, guerres, crises économiques...). Dans le cadre du projet ISyCri, nous nous intéresserons en priorité aux crises liées à des événements déclencheurs dont la survenue est brutale et par conséquent, à une mise en place rapide de la gestion de cette crise.

« Gérer» la crise est d'un certain point de vue une contradiction dans les termes. On ne gère pas l'évolution de la crise, on la subit. On s'efforce d'éviter que la situation ne s'aggrave et on essaie de rétablir l'ordre en tentant d'une part, de minimiser les effets identifiés et, d'autre part, de prévenir les risques de «surcrises » (http://fr.wikipédia.org/wiki/ Gestion_de_crise). Ainsi la littérature fait référence plus généralement aux termes de traitement d'une crise, de réponse à une crise (Lagadec, 1992 ; Devlin, 2006 ; Van Wassenhove, 2006). Une gestion de crise est généralement décomposée en quatre phases, pouvant coexister, décrites succinctement ci-après (Altlay et al, 2005 ; Beamon, 2004) :

1. Prévention. Cette étape consiste à traiter la vulnérabilité de l'écosystème par une gestion des risques permettant soit de diminuer la probabilité de survenue d'un événement déclencheur, soit de diminuer les conséquences de sa survenue (Beamon, 2004 ; Van Wassenhove, 2005).

2. Préparation. Cette étape consiste à prendre en compte les retours d'expériences afin de développer des nouveaux processus de réponses, d'une part, et de mettre en place l'organisation et les infrastructures qui permettront la mise en œuvre de la réponse à la crise, d'autre part.

3. Réponse. Cette phase consiste, lors de l'apparition d'une crise, à mettre rapidement en œuvre un processus de réponse faisant appel à des activités définies à l'étape précédente selon les caractéristiques de la crise.

4. Rétablissement. Cette phase consiste, en l'instauration d'une situation stabilisée et acceptable provisoirement (car bien souvent optimale compte-tenu des conditions et de la situation traversée), et gérer efficacement la transition vers le 
retour à la normale. Par exemple, cette phase peut concerner la reconstruction d'une zone dévastée, le suivi des personnes atteintes par la crise...

Dans la suite de cet article, nous nous intéresserons à la phase de réponse à la crise, correspondant à « l'ensemble des mesures prises pour résoudre des problèmes causés par la survenue d'une crise » (Devlin, 2006). La réponse à une crise dépend donc des caractéristiques de celle-ci. Par expérience, on sait que ces caractéristiques sont difficiles à identifier et par conséquent à modéliser. Par ailleurs, le traitement d'une crise est, par nature, un exercice délicat du fait de la variété des responsabilités engagées de la part d'acteurs hétérogènes devant travailler ensemble dans l'urgence (Hansen-Glize, 2008).

Lorsqu'une crise est déclarée, un collectif d'acteurs agissent ensemble dans le but de résoudre, ou au moins de réduire la criticité de la situation. Les points clés de cette prise en charge des problèmes sont essentiellement l'efficacité et la pertinence dont le collectif est capable de faire preuve. Il en découle une exigence forte sur la faculté des différents acteurs à collaborer efficacement durant le laps de temps où la crise est factuelle. Afin de répondre à cette attente, ce collectif s'organise de manière plus ou moins explicite ou formelle selon les types de crise. Nous émettons l'hypothèse qu'une cellule de gestion de crise est identifiée comme l'organe de pilotage qui doit faire face à la situation. Cette cellule de crise est soit unique (géographiquement ou au moins virtuellement), soit un membre indépendant d'un éventuel réseau de cellules de gestion de crise, en charge de missions spécifiques identifiées. Dans ce cas, le niveau d'indépendance requis pour chacune de ces cellules demeure cohérent avec l'étude dont il est ici question puisqu'il est associé à un comportement identifié qui pourrait être formalisé à l'aide d'un modèle de processus collaboratif, cœur de nos travaux. Notre étude se focalise sur le système d'information mobilisé par cette cellule pour gérer la collaboration et pour gérer les communications avec le terrain de manière bilatérale, qu'il s'agisse des remontées d'information permettant de suivre l'évolution des phénomènes, ou qu'il s'agisse de la transmission des consignes pour tenter de juguler le cours anormal des événements.

La caractérisation de l'espace collaboratif est marquée par deux traits principaux : (i) Un acteur qui entre dans la composition de la cellule de crise en tant qu'organisation souvent indépendante est dans une démarche coopérative, et il apporte des compétences au collectif. Il faut accepter un niveau d'autonomie de chaque acteur, et traiter avec lui en acceptant la spécificité de ses informations, de ses services et de ses processus. En effet, compte tenu de contraintes de durée et d'urgence tout autant que de la diversité incroyable des situations à imaginer, il n'est pas concevable d'imposer des standards opérationnels prédéfinis auxquels chacun devrait se conformer. La pression liée à une adaptation potentielle doit être, et doit rester, minimale. C'est un gage d'efficacité. (ii) Une crise est souvent un phénomène très dynamique, elle évolue au cours du temps et dans un univers qui est baigné d'incertitude. La capacité de prédiction avérée est en général assez faible. Ce constat induit un besoin de changer le spectre des compétences et/ou le champ de responsabilité d'acteurs au sein de la cellule de gestion de crise dans un mode plutôt 
réactif. La composition et les rôles du collectif de la cellule de gestion de crise sont donc eux aussi évolutifs. Dans l'idéal, pour être pertinent, les acteurs doivent quitter ou intégrer la cellule assez rapidement et à moindre effort.

La cellule de gestion de crise se doit donc d'être un système de pilotage ouvert et configurable pour faire front à une situation qu'on sait changeante. Il faut impérativement qu'elle se dote des moyens de surmonter des obstacles organisationnels et techniques qui sont souvent sans commune mesure avec la gravité des situations engendrées par la crise.

\subsection{Une exigence d'interopérabilité au cour de la problématique}

Nous proposons que ce problème soit formulé comme une exigence d'interopérabilité au sein de la cellule de crise. L'interopérabilité est appréhendée de manière large, incluant une dimension organisationnelle. La conséquence de cette position est que les solutions étudiées devront être inspirées par les travaux sur les architectures supportant l'interopérabilité entre des systèmes.

Il existe de nombreuses définitions de l'interopérabilité. Sur la base d'expériences vécues et d'une analyse de l'évolution des besoins en la matière à moyen terme, un des auteurs (Pingaud, 2009) a récemment proposé une définition adaptée à la problématique d'interopérabilité entre organisations : «l'interopérabilité désigne une capacité de systèmes, nativement étrangers les uns par rapport aux autres, à interagir afin d'établir des comportements collectifs harmonieux et finalisés, sans avoir à modifier en profondeur leur structure ou leur comportement individuel ».

Cette définition s'applique sans difficulté à la cellule de gestion de crise vue comme un système où les acteurs peuvent être mobilisés à la volée sans avoir eu le temps de se connaître mutuellement au préalable. Mais cette distance initiale n'est que théorique, elle sera vite effacée s'ils démontrent une capacité de couplage faible entre leurs systèmes, ce qui leur prodiguera ensemble une possibilité de bâtir un comportement collectif et harmonieux. Ce dernier but sera d'ailleurs symbolisé dans nos travaux par l'existence d'un processus collaboratif qui les réunit, qui rythme leur engagement et auquel ils s'attacheront à rester conformes Ce processus, qualifié de collaboratif puisque les activités qui le composent peuvent appartenir à des organisations différentes (Aubert et al, 2002), est bâti dans le but d'atteindre l'objectif commun de résoudre ou au moins réduire la crise. Il ne se limite donc pas seulement à coordonner les tâches des acteurs et à échanger de l'information. Il correspond à une coopération entre les acteurs selon les critères de (Bouzguenda, 2006). Ce processus collaboratif a pour but d'éviter les problèmes d'incompréhension, identifiés dans (Hansen-Glize, 2008), entre des acteurs poursuivant les mêmes objectifs de manière trop décorrélée. L'hypothèse de l'unicité de ce processus repose sur deux points : (i) l'étude ne se focalise que sur la dimension «opérationnelle » des processus du réseau des acteurs de la résolution de 
la situation de crise (les dimensions « pilotage » et « support », au sens de la norme ISO 9001:2000, sont laissées de côté au profit de la réalisation effective). (ii) la vision favorisée pour le processus collaboratif se base sur une proposition de séquence d'intervention nominale, favorisant le parallélisme des activités, sans alternatives identifiées puisque les ajustements sont laissés à la seule responsabilité de passage à un autre processus correspondant à une priorité d'agilité du système (cf. section 5). Ainsi, ce processus collaboratif évoluera structurellement à chaque événement marquant une modification significative de la situation, conjoncturelle (évolution qualitative ou quantitative de la crise) ou contextuelle (changement de fonction, de rôle ou d'acteur au sein de la cellule). Le processus collaboratif est donc assujetti à des phénomènes de reconfiguration au sein de l'organisation.

Une partie de la solution qui retient notre attention est donc un système d'information collaboratif basé sur cette idée de couplage faible. Il est ainsi décrit dans (Bénaben et al., 2007), comme un résultat du projet de recherches ISyCri (ANR-CSOSG) apte à satisfaire les exigences d'une cellule de gestion de crise.

\subsection{Plan de l'article}

Dans la première partie de cet article (cf. section 2), nous présentons, sous un nouveau format, les fondements de notre analyse des besoins. Pour cela, nous mobiliserons le concept de système de systèmes $(\mathrm{SdS})$ pour synthétiser de façon très générique les attendus des métiers d'une cellule de gestion de crise. Nous montrerons que la structure d'un système de systèmes offre naturellement un cadre conceptuel qui favorise la formulation du problème d'architecture de système d'information dans le cadre des caractéristiques dominantes du cas d'étude (cf. section 3). Un système de systèmes confine les choix de conception au niveau des interfaces des systèmes contributeurs puisque l'adaptation de ces entités, si elle est possible, ne peut pas être synonyme de profonde mutation. Dès lors, le lien avec les capacités en interopérabilité des partenaires sont établis.

Puis, nous argumenterons sur l'existence d'un système tier, un système de médiation, au centre du dispositif. Ce nouveau venu doit prendre en charge le collectif et favoriser une forme d'agilité entre l'espace de collaboration métier et son système d'information collaboratif. Pourquoi ce nouveau système en gestion de crise ? En matière de comportement, l'efficacité et la traçabilité des échanges entre les partenaires de la cellule de gestion de crise ne sauraient se satisfaire d'une organisation basée sur des échanges de type point à point (cf. figure 1, cas(a)). Dans le même ordre d'idée, la distribution des compétences et le maintien de l'autonomie des contributeurs sont deux facteurs qui ne militent pas pour un pilotage trop centralisé dans lequel un système de contrôle serait amené à prendre une responsabilité hiérarchique couvrant à lui seul l'ensemble des fonctions attendues (cf. figure 1, cas(b)). Nous avons donc opté pour une solution à mi-chemin entre ces deux extrémités. Le concept de système de médiation est au centre d'un dispositif 
qui donne naissance successivement au système d'information de médiation (SIM), puis à une solution logique via un composant médiateur.

C'est une déclinaison particulière de l'architecture de médiation promue par (Wiederhold, 1992) dans un contexte de recherche d'informations, avec l'idée de fédérer des ressources informationnelles distribuées. Notre architecture propage cette idée au niveau des connaissances sur les métiers. Le but est de savoir coordonner les activités des contributeurs en imposant une structure de contrôle dictée par des processus collaboratifs qui doivent être exécutés avec conformité. Le système de médiation est immergé au sein du système de systèmes (cf. figure 1, cas(c)).

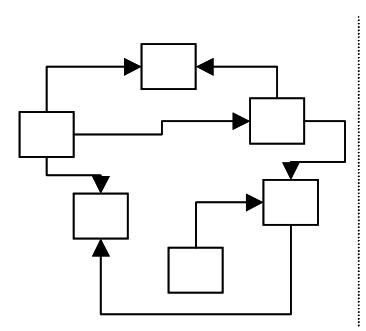

(a)

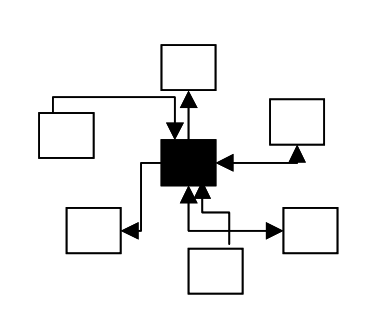

(b)

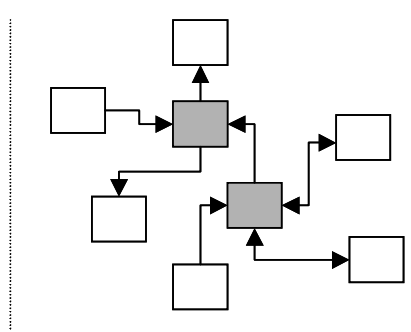

(c)

Système contributeur de la gestion de crise

Système de contrôle centralisé

$\longrightarrow \quad$ Flux d'information

Figure 1. Plusieurs formes d'architecture du système de systèmes

L'article prolonge donc cette vision SdS en se focalisant sur la conception de systèmes d'information de médiation (SIM) adaptés à la crise, et qui vont réunir autour d'eux les systèmes d'information contributeurs (cf. section 4). Nous ne présentons pas ici la partie décisionnelle du système de médiation. L'aide à la décision pour le choix de politiques de gestion de crise (résolution de crise, définition de scénarios...) est un sujet intéressant qui fait l'objet de travaux complémentaires dans le projet ISyCri. Le système d'information médiateur est le centre nerveux du système d'information collaboratif. C'est sa contribution dans l'architecture globale du système que nous décrivons dans la seconde partie de l'article. Il doit assurer des compatibilités entre des parties publiques des systèmes contributeurs communicants, et le système qu'il représente doit assumer les obligations de coordination des acteurs via un processus collaboratif cible. Le composant médiateur, qu'il inclut à un niveau logique, est assimilable à une collection de services en interopérabilité, telle qu'elle définie dans certains projets en interopérabilité d'entreprise qui font l'hypothèse d'une architecture orientée services (Roadmap EI, 2008). 


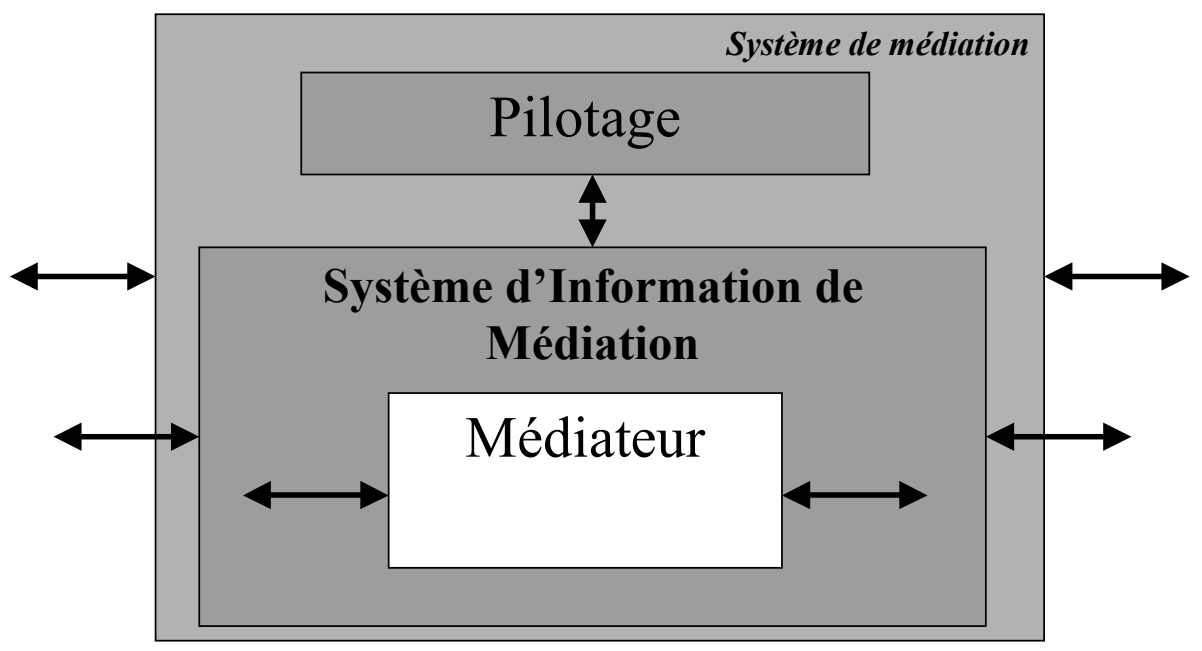

Figure 2. Structure du système de médiation

Cette architecture du système d'information collaboratif basée sur la médiation sera illustrée par un cas d'études : une simulation d'accident nucléaire, radiologique, biologique et chimique (NRBC) est réalisée par la préfecture du Tarn (81). Au moyen de ce cas d'études, nous expliquerons différentes phases d'ingénierie dirigée par les modèles qui ponctuent la mise au point du médiateur au sein d'un bus de services d'entreprise. Pour cela, nous partirons de la connaissance disponible en ligne sur la situation, et de la connaissance disponible sur les compétences des partenaires pour bâtir une solution au niveau métier pour le collectif.

Dans un troisième temps de cet article, nous traiterons plus spécialement une forme d'agilité dont il est possible de faciliter le développement pour faire face à l'organisation changeante de la cellule de crise (cf. section 5). En effet, les motivations pour le développement d'une interopérabilité peuvent être classées en trois natures de changement au sein d'un réseau (Pingaud, 2009) :

(i) variations dans la mission d'un réseau,

(ii) variations dans la structure des systèmes contributeurs ou du réseau,

(iii) variations du comportement dans les échanges au sein du réseau.

Reprenant cette classification, nous la transposerons à des évolutions de la gestion de crise où l'agilité du système est mise à contribution sous le coup d'événements qui modifieront un mode de collaboration comme :

- des variations des caractéristiques perçues de la crise, correspondant à la variation (i), 
- des variations du pilotage de la résolution de la situation de crise par la cellule de gestion de crise, correspondant aux variations (ii) et (iii).

\section{Une vision inspirée du concept de système de systèmes}

Il existe plusieurs définitions du concept de système de systèmes. Récemment, un ouvrage de synthèse produit par des auteurs en ingénierie des systèmes (Luzeaux et al., 2008) en a fait la synthèse et a proposé la définition suivante :

« Un système de systèmes est un assemblage de systèmes pouvant potentiellement être acquis et/ou utilisés indépendamment, pour lequel le concepteur, l'acquéreur et/ou l'utilisateur cherche à maximiser la performance de la chaîne de valeur globale, à un instant donné et pour un ensemble d'assemblages envisageables ».

On retrouve dans cette définition une forme très condensée des différences entre le concept de système de systèmes, et celui de système. Ces différences sont qualifiées par cinq critères discriminants proposés par (Maier, 1998) : (i) Indépendance opérationnelle des éléments (pouvant être des systèmes), (ii) Indépendance managériale des éléments, (iii) Développement évolutif, (iv) Comportement émergent, (v) Distribution géographique.

Examinons ces différents critères dans le cadre d'une réponse à une crise.

Les acteurs doivent unir leurs efforts et interagir, avec deux finalités principales :

- comprendre la crise, la suivre et la caractériser,

- agir pour tenter de résoudre, ou tout au moins de réduire, la crise. Les actions entreprises par les systèmes contributeurs sont de nature préventive ou curative.

Cependant, ces acteurs ne sont pas nécessairement placés sous une seule et même autorité (critère(i)). Si cela est vrai dans le cas d'une crise civile (plan orsec, plan rouge...) en France, par exemple, cas où les préfets de département ou de région sont en responsabilité avec une situation de leader ayant pour obligation de déployer le plan selon des procédures (i.e. des processus) prédéfinies, cela n'est pas aussi net dans le cas d'une crise humanitaire quand des acteurs divers tels que l'ONU, des institutions nationales et des organisations non gouvernementales multiplient les actions assez individuellement, sur une base de compétences dont la complémentarité est à évaluer. Ils sont ainsi, de fait, en cogestion de crise, partageant les informations pour construire une coordination qui est conçue en situation sur le terrain (critère(ii)).

En somme, l'hétérogénéité des acteurs et leur autonomie de management sont généralement reconnues comme un fait réel pour ce type de situation. Chacun prend les responsabilités qui sont les siennes dans son champ de compétences. Par conséquent, la gestion de la crise peut être vue comme un ensemble de plusieurs systèmes autonomes qui doivent collaborer, à la fois pour la prise de décision et pour 
les opérations. Selon la crise et son degré d'expansion, différents systèmes peuvent intervenir dans le but de la résoudre. Nous pouvons aussi considérer qu'il peut y avoir une distribution géographique des systèmes quand la crise concerne un territoire étendu, dépassant des limites d'un pays et de son gouvernement, par exemple (critère (v)). La nature évolutive de la crise a des répercutions sur la composition de l'ensemble des acteurs qui doivent intervenir ainsi que les services qu'ils rendent pour la résoudre. La nature potentiellement évolutive d'une crise est donc confirmée (critère (iii)). Enfin, si les acteurs se concertent afin de jouer sur la complémentarité de leurs compétences, la gestion de la crise ne peut qu'être bénéficiaire. Par conséquent, la gestion de la crise a probablement un comportement émergent (critère iv).

Nous pouvons donc conclure que la gestion de crise a toutes les caractéristiques spécifiques d'un système de systèmes. Parce qu'ils forment un sous-ensemble de ce $\mathrm{SdS}$, les systèmes d'information des différents acteurs doivent interagir pendant la gestion de la crise pour informer sur son évolution autant que pour engager des actions préventives ou curatives de traitement des risques.

\section{Une architecture à trois niveaux}

Notre proposition d'architecture du système d'information de gestion de crise suit un modèle en trois couches : métier, logique et technique.

La couche métier décrit les besoins et les usages attendus du système. La mise en relation appropriée des acteurs y est un point critique. La couche logique explique la structure des composants applicatifs mis en œuvre pour supporter la couche métier, ainsi que les formes de relation qui s'établissent entre ces composants. La couche technique explique comment les composants applicatifs sont déployés et utilisés sur le matériel mis à disposition.

Un système d'information de médiation (SIM), comme expliqué dans (Touzi et $a l .$, 2008), insère un composant intermédiaire entre les systèmes contributeurs. Ce médiateur peut être perçu comme un média capable de supporter et faciliter la collaboration entre les partenaires. Sa conception doit effacer toute forme de résistance. Il doit pouvoir se connecter à l'ensemble des systèmes d'information des partenaires, traduire les données afin de les échanger, exécuter les services selon la prescription fournie par un processus. Il s'agit bien d'un système d'information spécifique puisqu'il fait intervenir des acteurs (les systèmes contributeurs) qui interagissent selon le processus collaboratif qui véhicule des flux d'information entre les services (cf. figure 2). Qui plus est, aux éventuelles hypothèses d'architecture pear-to-pear ou de distribution chez les différents partenaires des fonctions support de l'interopérabilité (a minima: le transfert d'information, la gestion du partage des fonctionnalités et le pilotage du comportement collaboratif), nous opposons une explosion combinatoire «victime» d'une nécessaire nonstandardisation des formats de données, des protocoles d'accès au services et d'orchestration des workflows). Ainsi, l'adjonction d'un SI de médiation, au sein 
d'une communauté de SI collaboratifs, semble la solution la plus raisonnable pour supporter l'interopérabilité des partenaires. Ce SI de médiation s'il devient incontestablement la composante cruciale du bon comportement du réseau d'organisation, n'en est pas pour autant critique quant aux partenaires eux-mêmes. Une hypothétique disparition n'entraînerait qu'un retour du système de systèmes à un ensemble de systèmes, indépendants mais intacts dans leur connaissance, leurs compétences et leur savoir-faire. Nous n'oublions pas les difficultés liées aux différences sémantiques dans l'identification des services et dans les interprétations de données. Elles ne font pas l'objet d'un développement dans cet article. Le problème est formulé dans le cadre de travaux menés en parallèle aux nôtres, et fait l'objet de recherches particulières pour étoffer le médiateur (Rajsiri, 2009).

Revenant au concept de système de systèmes, les systèmes contributeurs seront appréhendés par les fonctions qu'ils remplissent pour le collectif, et donc par les services qu'ils lui rendent. Ces systèmes seront divisés en deux parties : une partie publique où toutes les entités nécessaires à la collaboration (processus, applications et données) seront accessibles, et une partie privée en charge de garantir l'expression des compétences d'un système contributeur dont la visibilité peut être réduite au maximum dans le dessin de l'architecture.

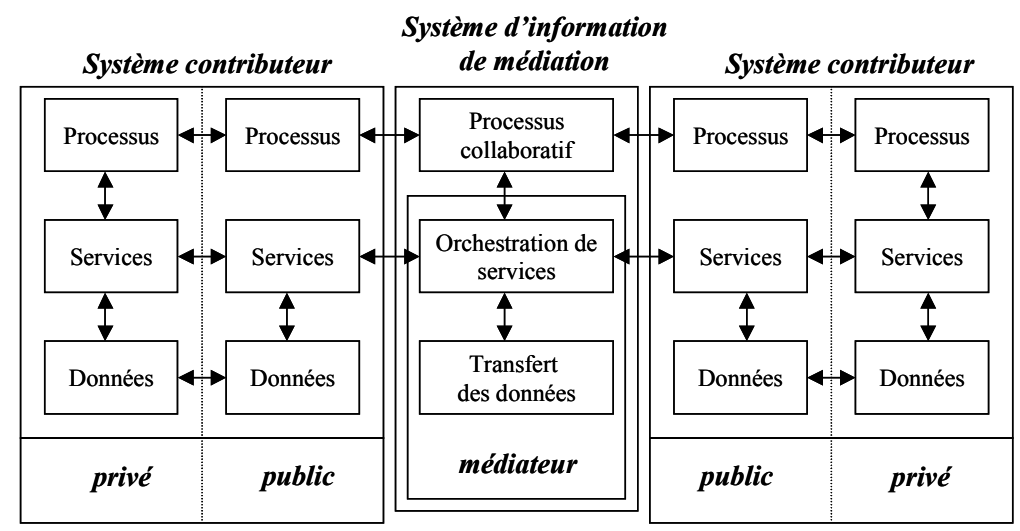

Figure 3. Le système d'information de médiation lie les parties publiques des systèmes contributeurs (relations entre couche métier et couche logique)

$\mathrm{Au}$ sein des systèmes contributeurs, les processus fournissent la structure de contrôle pour l'invocation des services applicatifs. De nombreux auteurs utilisent le concept de services métiers pour indiquer les points de connexion avec ces processus métiers de chaque contributeur. Les services applicatifs accèdent à leurs données propres lors de leur exécution, souvent par de simples accès aux bases de données. 
Au sein du système d'information de médiation, le processus collaboratif peut être apparenté à un modèle de workflow, puisqu'il offre une structure de contrôle de tout ou partie de processus communicants entre des systèmes contributeurs. Il fournit donc les directives au moteur d'orchestration qui a en charge l'exécution de ce processus collaboratif via les invocations de services des systèmes contributeurs. Enfin des services ad hoc s'occupent du transfert des informations entre systèmes contributeurs.

Pour la couche logique, nous avons fait ce choix d'une architecture orientée services (SOA) parce qu'elle offre naturellement des avantages pour développer des systèmes à base de composants applicatifs faiblement couplés. L'approche par médiation s'accorde bien avec un choix SOA. En effet, le médiateur est alors assimilable à un rôle de courtier des services offerts publiquement par les partenaires. Il en a connaissance grâce au registre collectif des services dont il a la charge et qu'il entretient.

$\mathrm{Au}$ niveau technique, nous avons bâti notre proposition en mobilisant une technologie de bus de service d'entreprise (ESB ou Entreprise Service Bus). Une solution du monde du logiciel libre, l'ESB PEtALS, a été choisie comme plateforme de développement. PEtALS est un produit du consortium ObjectWeb (http://petals.objectweb.org ) dont la diffusion est assurée par une jeune société partenaire du projet ISyCri (EBM WebSourcing). Le choix de l'ESB se base sur sa capacité à offrir une solution technique offrant, en un tout cohérent, tous les moyens nécessaires au déploiement. En effet, (i) la plateforme utilise les standards et les principes architecturaux du monde SOA, (ii) une fois connecté au bus, un service applicatif est connecté à l'ensemble des autres services, (iii) un service d'orchestration, appelé Orchestra dans PEtALS, prend en charge la séquence d'appels des services pour suivre un processus de traitement préspécifié, i.e. il gère l'appel des services dans un ordre précis et selon certaines règles. C'est donc au sein même de l'ESB que l'on retrouve les moyens de déployer les deux fonctions principales du médiateur.

\section{Présentation de la démarche de conception par un cas d'étude}

Le modèle en trois couches présenté en section précédente correspond point par point aux trois niveaux définis par l'OMG dans son architecture dirigée par les modèles (MDA). La couche métier doit correspondre au niveau CIM (Computer Independent Model). La couche logique correspond au niveau PIM (Platform Independent Model). Enfin, la couche technique correspond au niveau PSM (Platform Specific Model).

Déclinons maintenant ce modèle architectural sur une problématique de gestion de crise afin d'identifier ce que sont les modèles correspondants. La couche métier utilise des connaissances relatives à la crise, d'une part, et une connaissance des rôles joués par les acteurs à travers les compétences publiées qu'il faut réunir (que nous pourrons appeler services métiers), d'autre part. Cette connaissance sur les services métiers est construite à partir des résultats de la phase de préparation de la 
gestion de crise. Par exemple, pour les crises civiles les différents plans (Plan Orsec, Plan Blanc, Plan Rouge...) et les fiches réflexes associées constituent le principal « capital » de connaissance issu de la phase de préparation.

Dans notre solution, les informations sont injectées dans une ontologie de crise qui permet de déterminer, par croisement entre les deux formes de connaissance, les services métiers pouvant être utilisés dans le cadre de la réponse à la crise. Comme expliqué dans (Truptil et al., 2009), ce croisement se base sur la définition des services métiers qui sont caractérisés part quatre relations : (i) prévenir un risque, (ii) réduire une conséquence (faits avérés de la crise), (ii) nécessiter l'exécution d'un service avant ou (iv) après. Sur la base de ces relations et de la connaissance de la crise, des règles écrites en SWRL (Semantic Web Rule Language), disponibles dans le même article, sont exécutées automatiquement par un moteur d'inférence Jess (http://www.jessrules.com/jess/).

A l'issue de cette déduction, et pour chaque caractéristique de la crise (à savoir les faits significatifs de la situation de crise, mais également les risques associés à cette même situation), la cellule de crise se voit proposer l'ensemble des services métiers (identifiés dans l'ontologie) pouvant être utilisés à l'encontre de cette caractéristique (prévention de risque ou traitement d'un fait). La cellule de crise doit alors, d'une part, sélectionner pour chacune de ces caractéristiques l'ensemble des services métiers adéquats, et, d'autre part, définir la priorité de traitement de ces caractéristiques. Une seconde vague de règles de déduction, également décrite dans (Truptil et al., 2009), est alors exécutée. Cette seconde vague est constituée de deux types de règles: tout d'abord, (i) pour chaque service métier sélectionné (ou étant nécessaire à un autre service), un service de médiation est créé afin de pouvoir transmettre et recevoir des informations pour l'exécution du service métier. Ensuite, (ii) il s'agit de définir l'ordre d'exécution des différents services : cet ordre se base sur la priorité de traitement des caractéristiques de la crise, i.e. les services métiers utilisés pour résoudre la caractéristique la plus critique seront les premiers à être exécutés. Cependant, nous supposons que si deux services ne traitent pas le même problème, n'interviennent pas sur la même partie de l'écosystème et ne sont pas réalisés par le même acteur alors ils peuvent être mis en parallèle. Concrètement, cela signifie que la deuxième vague de règle ambitionne de paralléliser un maximum d'activités (services métiers), et, lorsque ceci s'avère impossible, les critères d'ordonnancement s'appuient alors sur la hiérarchie de criticité établie au sein de la cellule de crise.

Le résultat de ces règles se concrétise par la déduction d'un processus collaboratif. Ce processus collaboratif, qui est un résultat tangible du raisonnement sur la connaissance, est ensuite validé par la cellule de crise et mis à disposition explicitement sous forme d'un modèle en langage BPMN relatif à la couche « métier». Cette mise à disposition automatique est réalisée par le biais de l'outil ATL et d'une transformation automatisée de modèles (Rajsiri, 2009). Il concentre l'essentiel de la spécification pour la conception du médiateur. 
Afin d'illustrer cette méthode de conception, nous introduisons un exemple basé sur un exercice de réponse à une crise de type NRBC (nucléaire, radiologique, biologique et chimique) exécuté par la préfecture du Tarn (81).

Le scénario joué est le suivant : « Le 27 février 2004 à 10h du matin, la gendarmerie est informée qu'il s'est produit un accident entre un camion citerne et un wagon contenant des produits chimiques inconnus qui s'échappent dans l'atmosphère. De plus, les gendarmes envoyés sur les lieux ainsi que les employés de la gare sont inconscients. Enfin, les enfants présents dans la cours de l'école proche de l'accident sont saisis de malaises. »

Le modèle présenté en figure 4 est le modèle de crise de cet exemple NRBC. On y retrouve l'événement «collision » qui déclenche la crise, le risque d'explosion ainsi que les conséquences potentielles émanant d'une analyse préliminaire des risques. Le dégagement de produit chimique est effectif et des personnes sont malades (le personnel de la gare, les gendarmes et les enfants de l'école). Le dégagement de produit chimique engendre le risque de contamination qui menace l'environnement (sites naturels). De plus, le fait que l'accident se soit déroulé à proximité de l'école peut engendrer un autre risque de panique au niveau des parents qui voudraient absolument récupérer leurs enfants.

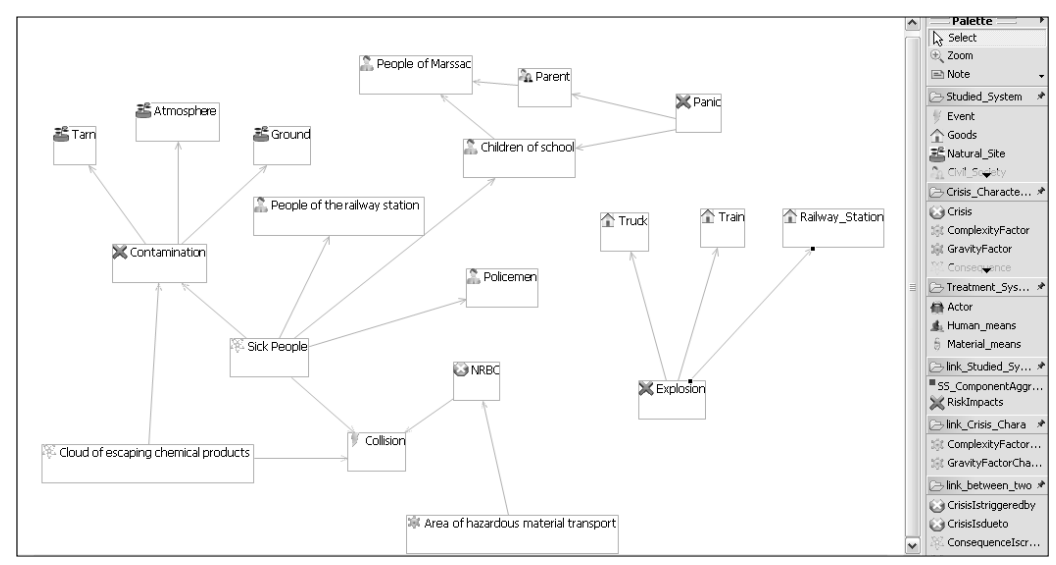

Figure 4. Modèle de crise NRBC (IsyCrisisTool)

Nous avons mis au point deux composants logiciels IsyCrisisTool et IsyServiceTool dotés d'interfaces permettant de saisir les informations courantes sur la crise pour le premier, et sur les compétences des partenaires impliqués pour le second. En particulier, un langage adapté à la caractérisation de crise a été mis au point via un métamodèle (palette de droite de l'outil IsyCrisisTool, figure 4) (Truptil et al., 2008). 
Une fois ces informations saisies, elles sont injectées dans une ontologie de domaine, nommée OntoServiceState et en deviennent des instances. Des règles de déduction sont appliquées sur ces instances afin de déterminer l'ensemble des services métiers utilisables lors de la réponse à la crise. Cet ensemble est ensuite publié.

Dans l'exemple, il a été décidé de traiter les problèmes dans l'ordre suivant :

- réduire le risque d'explosion,

- réduire le risque de contamination,

- s'occuper des personnes malades,

- éviter le risque de panique,

- trouver des moyens supplémentaires pour décontaminer l'ensemble des personnes.

Les autres risques présents ne sont pas jugés prioritaires, ils peuvent exister, mais il a été décidé qu'il n'y aurait pas de mesure prise pour traiter ces risques, dans un premier temps.

La cellule de gestion de crise prend alors la main car la partie caractérisation s'achève, et il faut prendre des décisions sur les actions à mener pour les systèmes contributeurs (dans l'exemple: compétences de Sécurité civile, gendarmerie, SAMU, Croix Rouge). L'ordre de priorité dans le traitement de la crise, ainsi que l'ensemble des services utilisés lors du processus de réponse à la crise, sont établis. Les responsables ont ainsi décidé d'utiliser les services suivants pour maîtriser la crise (l'ordre d'exécution des services correspond à la numérotation et l'acteur qui en est responsable figure entre parenthèse) :

- 1 : FightExplosion (pompier), SetSecurityPerimeter (gendarmes).

- 2 : HowmanyPeopleToRescue (pompier), SetEquipement (gendarmes).

- 3 : RescuePeople (pompier), MaintainPerimeter (gendarmes), SetMedicalPost (Croix Rouge).

- 4 : BringPeopleToMedicalPost (Croix Rouge).

- 5 : Care People (SAMU).

Cet ensemble donne naissance au processus collaboratif qui est soumis à validation par la cellule avant d'être lancé. A la fin de cette phase, le modèle CIM au format BPMN, est alors disponible (cf. figure 5).

Ce processus collaboratif respecte un métamodèle de processus collaboratif dans lequel le système d'information de médiation est symbolisé par une ligne d'eau et apparaît donc comme un acteur du système. C'est la seconde ligne du modèle (figure 5), on voit qu'elle seule utilise des liens de précédence et des opérateurs logiques. C'est dans cette ligne qu'est la structure de contrôle du processus collaboratif. Ce modèle de niveau CIM est ensuite transformé en modèle UML du médiateur avec un profil SOA adapté (PIM4SOA, 2005) par une transformation 
expliquée dans (Touzi, 2007) et déployée avec un outil de transformation de langage (Jouault, 2006).

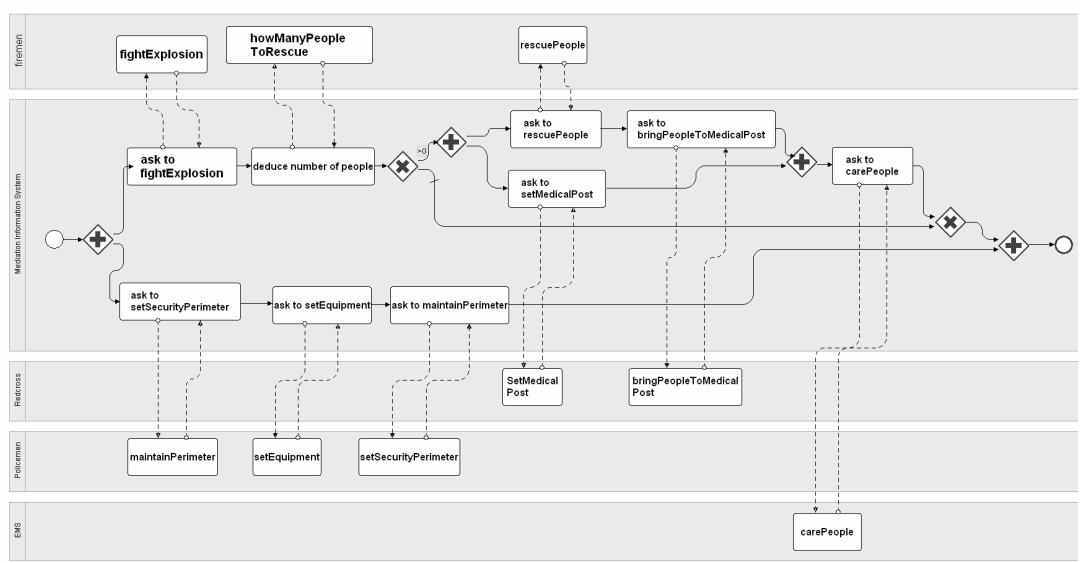

Figure 5. Processus collaboratif de réponse à la crise NRBC

Cette architecture logique est ensuite mise à profit afin de terminer le déploiement du médiateur. Un fichier au format BPEL sert de point d'entrée pour le moteur de workflow Orchestra de PEtALS. Ces deux dernières étapes sont transparentes pour les décideurs car elles sont automatisées. Au final le résultat est résumé sur la figure 6 .

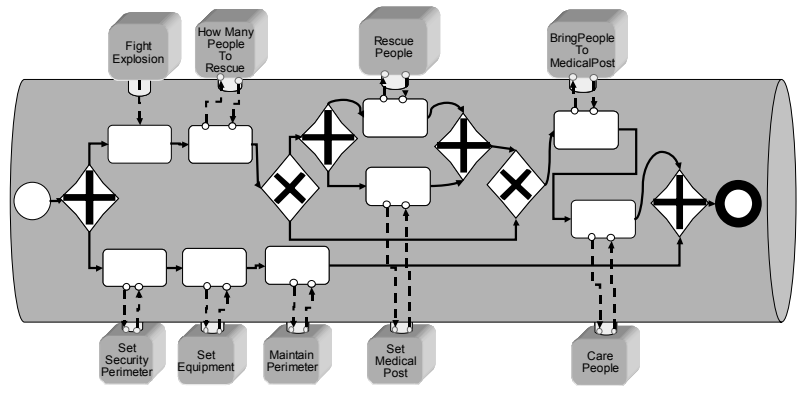

Figure 6. Représentation schématique du médiateur obtenu dans l'ESB pour l'exemple de la crise $N R B C$

Le processus de conception du médiateur est résumé sur la figure 7. Une lecture du haut vers le bas permet de visualiser l'enchaînement des étapes du processus d'ingénierie dirigée par les modèles à chaque niveau d'abstraction du MDA. 


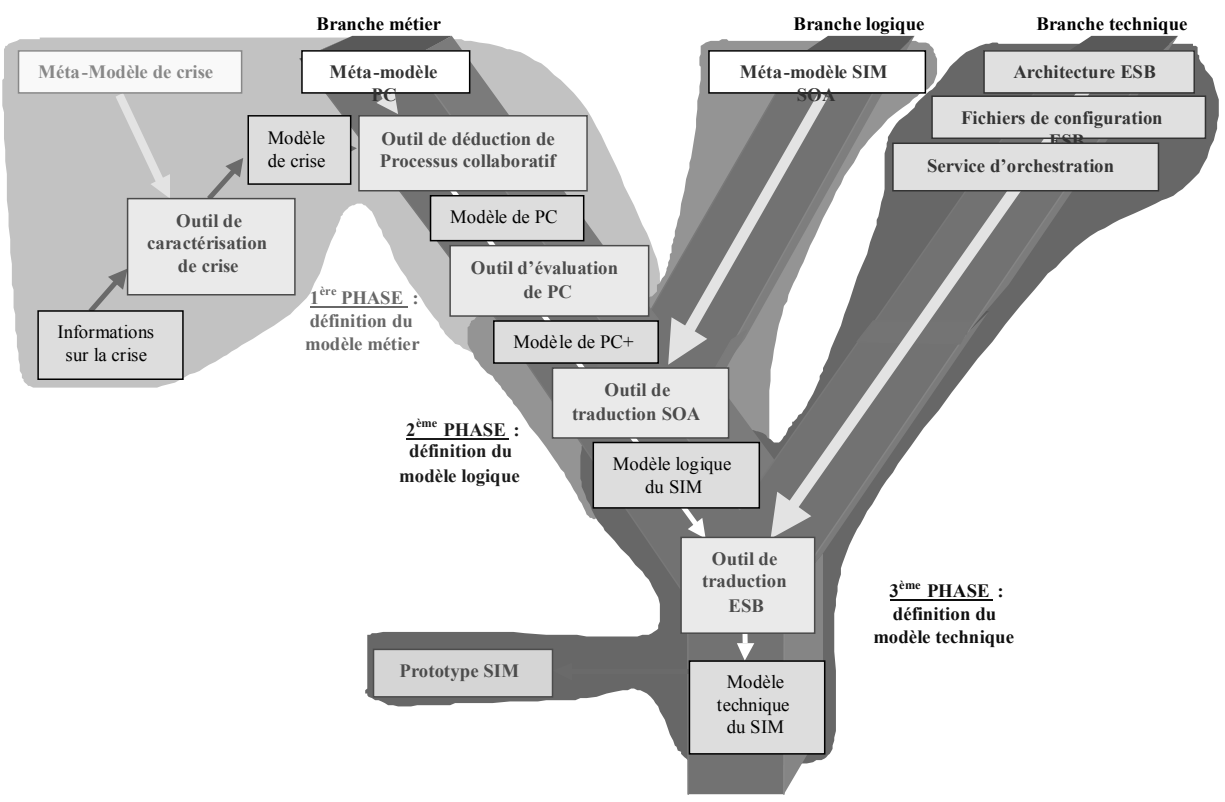

Figure 7. Vue d'ensemble de la démarche de création du SIM

Nous venons d'avoir une vue d'ensemble de la démarche de création du système d'information médiateur. Dans le contexte de gestion de crise, il est évidemment indispensable que cette création soit rapide. Nous avons vu qu'une fois que le processus collaboratif est défini, la suite de la démarche est totalement automatisée. Le critère de rapidité de mise en place du SIM dépend donc essentiellement du temps de création du processus collaboratif. Or celui-ci est construit à partir (i) des informations relatives aux services métiers définies lors de la phase de préparation à la gestion de crise, et par conséquent avant son apparition, (ii) de la caractérisation de la situation de crise effectuée dès l'occurrence de cette dernière (à partir des informations recueillies et (iii) de la priorisation des faits et risques identifiés pour la crise et la sélection, parmi ceux proposés automatiquement, des services (curatifs ou préventifs) associés. La rapidité de construction du SIM dépend alors des deux derniers items identifiés, caractérisation puis priorisation/sélection. Concernant le premier point (la caractérisation de la situation de crise), il semble évident de noter que quel que soit le mode de fonctionnement de la cellule de crise (avec ou sans médiateur), une phase de prise de connaissance et d'assimilation de la situation semble incontournable. La capitalisation de cette connaissance sous la forme d'un modèle formel (tel que proposé par notre approche) ne constitue pas une activité chronophage susceptible de déséquilibrer cette phase d'analyse de la crise. Nous proposons donc de conclure que l'activité de caractérisation de la crise, si elle ne raccourcit pas la phase d'acquisition de connaissance sur la situation, n'est pour 
autant nullement responsable d'un rallongement critique. Concernant le dernier point (priorisation/sélection) ces choix peuvent évidemment être effectués très rapidement comme très lentement. Néanmoins, ces choix pouvant, à n'importe quelle étape de la réponse à la crise, être remis en cause et modifiés grâce à l'agilité de la démarche, nous pouvons imaginer que la cellule de crise, rassurée par cette souplesse, sera, si ce n'est plus prompte, tout du moins aussi rapide qu'elle peut l'être aujourd'hui pour décider des actions à mener. Enfin, les tests sur les cas d'usage et les scénarii de démonstration relatifs au projet ISyCri, ont montré que les opérations automatisées de déduction et de transformation de modèles nécessitaient des temps très faibles, toujours inférieurs à la minute.

\section{Vers une démarche de détermination du SIM agile}

La section précédente présente la démarche de conception d'un système d'information médiateur (SIM). Nous avons pu voir que cette démarche repose sur un unique processus collaboratif de réponse correspondant à un scenario possible de résolution de la crise. Cette vision contraste avec celles qui proposent de définir un certain nombre de processus de réponse possibles et d'y apporter des modifications dynamiquement afin de réagir à des événements de la crise, comme les travaux de (Rueppel et et al, 2007) concernant les réponses aux inondations en Allemagne. Bien que cette vision soit intéressante et présente certains avantages, nous supposons qu'il est difficile de connaître a priori l'ensemble des événements potentiellement susceptibles de se produire, pouvant non seulement modifier la gravité de la crise mais aussi sa nature. L'inhérente instabilité et imprévisibilité des situations de crise en général, restreint ces approches à des domaines de crise bien particuliers, identifiés et bien maîtrisés (qualitativement et quantitativement). Dans bien d'autres crises, ce degré d'anticipation n'est pas possible. Qui pouvait anticiper le basculement de la situation de canicule de l'été 2003 d'une crise sanitaire à une crise sociale d'une telle ampleur? Ces considérations justifient, d'une part, la volonté affichée dans cet article de ne pas essayer de "cartographier» a priori, l'espace des situations de crise et l'espace de la gestion de crise, (ce qui nous semble utopique dans ces environnements à l'instabilité chronique) et, d'autre part, l'incontournable nécessité de pouvoir remettre en cause, sur toute évolution, conjoncturelle ou contextuelle de la situation de crise, la dynamique de réponse construite. Cette nécessaire agilité du SI de médiation doit permettre à la cellule de crise de demeurer pertinente quant à la gestion de la situation.

\subsection{Définition de l'agilité}

L'agilité est devenue une propriété très étudiée depuis la fin des années 1990. Elle prend un sens particulier lorsqu'elle qualifie une collaboration interentreprises. Cet usage a été décrit dans (Bénaben et al., 2007) comme «la transformation de la 
structure figée vers un environnement fluide», mais elle est aussi décrite dans (Luzeaux et al.,2008) avec une autre image, comme «la transformation d'une construction statique de lego® vers un organisme vivant». Ces métaphores sous entendent qu'aujourd'hui les entreprises ne collaborent plus nécessairement dans un optique de long terme, elles cherchent des moyens pour collaborer ponctuellement sur des horizons de temps moins longs. Il y a un parallèle entre ces analyses d'écosystèmes industriels et notre application plus orientée vers le monde des services. Une problématique d'aide à la gestion de crise ne fait qu'amplifier le besoin d'agilité, qui n'est pas seulement recherchée mais inscrite dans les exigences.

Pour mieux cerner ce qu'agilité veut dire, nous avons utilisé des éléments d'une synthèse bibliographique sur l'agilité des entreprises. L'ensemble des définitions du concept d'agilité s'accordent sur le fait qu'une entreprise peut être dite agile si elle peut s'adapter à l'évolution du contexte dans lequel elle intervient. Certains auteurs comme (Badot, 1997) parlent plutôt de reconfiguration du système pour qualifier un besoin d'adaptation. D'autres auteurs, comme (Kidd,1994), (Lindberg,1990) et (Sharifi et al.,1999) parlent plutôt d'une aptitude à satisfaire un changement dans un temps court pour qualifier un besoin de réactivité. Nous avons décidé, dans le cadre du projet ISyCri, de décliner la notion d'agilité en retenant ces deux dimensions largement partagées:

- la réactivité se concentre sur le rapport au temps, nous allons faire l'hypothèse que ce temps est un délai court qui n'accepte des modifications qu'au seul niveau des services. La réactivité peut être définie comme la capacité d'un système d'information collaboratif d'être rapidement opérationnel suite à une demande de modification d'une fonction dévolue à un acteur connu, la structure de contrôle du processus collaboratif n'a pas à être modifiée, le médiateur n'est pas obsolète,

- la flexibilité renvoie à une adaptation plus profonde. Elle doit permettre une mise en adéquation du système d'information collaboratif suite à des variations qui peuvent être : (i) des variations des caractéristiques de la crise induisant une reprise en totalité de la chaîne de conception depuis le modèle de la crise, (ii) des variations dans le mode de pilotage de la réponse à la crise à modèle de crise constant, c'est le processus collaboratif qui est alors modifié.

Cette décomposition nous permet de dissocier plusieurs catégories de problèmes selon le niveau d'impact de la variation sur le processus de réingénierie du médiateur décrit sur la figure 7. Faut-il reprendre la configuration du système d'information de médiation au niveau du modèle de crise, au niveau du modèle de PC ou au niveau du modèle logique de SIM ?

\subsection{Développer l'agilité au niveau de la démarche de conception}

L'acquisition d'une capacité de réactivité n'est pas un obstacle majeur dans la mesure où la démarche de conception du système d'information collaboratif est systématique dès que le processus collaboratif est défini. En effet, puisque les 
systèmes d'information des systèmes contributeurs sont stables, le passage à l'acte consiste à actualiser l'adresse d'un ou plusieurs service(s) dès que la cellule de gestion de crise a validé le processus collaboratif actualisé.

Par contre, la flexibilité induit un traitement beaucoup plus lourd, comme le montre la figure 8 , toute la chaîne de conception du médiateur peut être touchée et relancée en vagues successives si la crise évolue très rapidement.
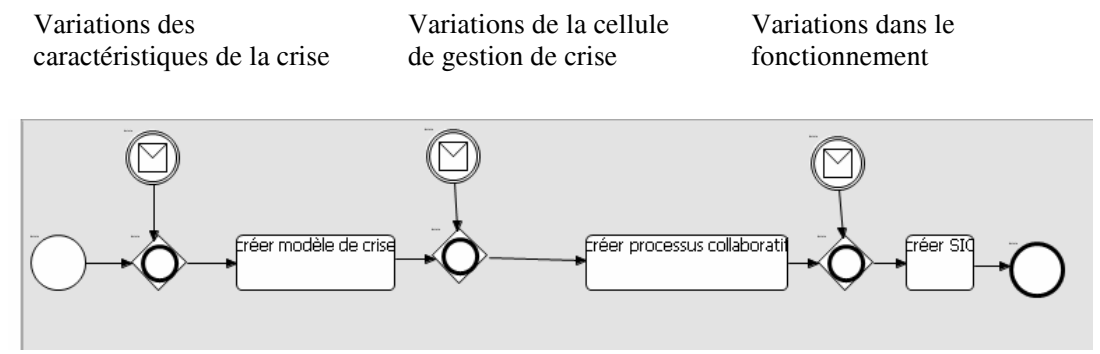

Figure 8. Vue simplifiée du processus de conception avec la prise en compte des variations

Afin de faciliter la mise en œuvre d'une rétroconception récurrente $\mathrm{du}$ médiateur, nous avons imaginé d'embarquer l'ensemble des outils de notre chaîne de conception du médiateur, telle qu'elle apparaît sur la figure 7, sous forme de services dans l'ESB PEtALS. La figure 9 représente cette solution de façon schématique.

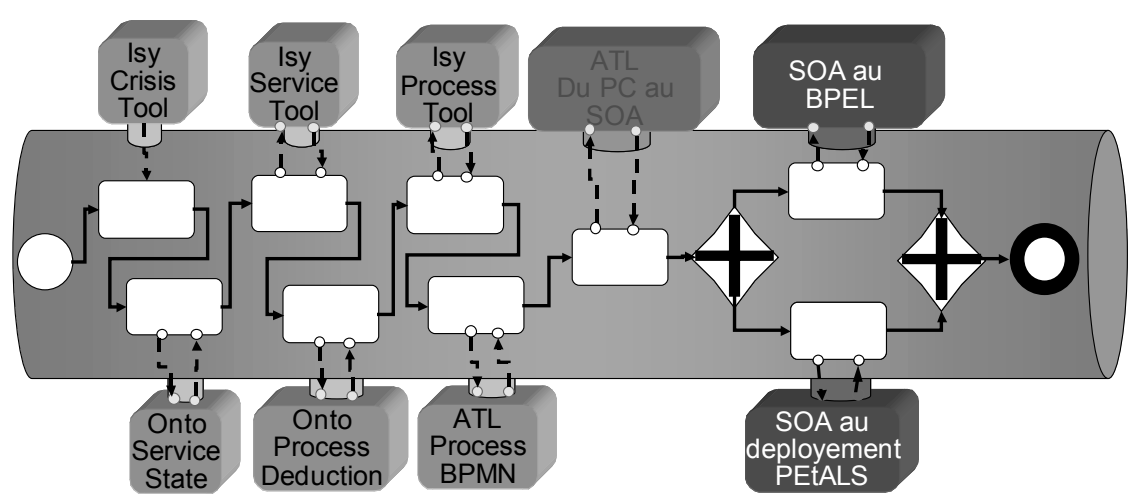

Figure 9. Vers une démarche de conception flexible du médiateur 


\subsection{La fiche de suivi}

Pour détecter qu'une variation a lieu, une fiche synthétique de suivi de la crise est proposée. Une collecte des éléments d'information est opérée sur plusieurs plans : l'évolution des caractéristiques de la crise, l'évolution de l'exécution des services des acteurs ainsi que du groupe d'acteurs eux-mêmes. Cette opération s'apparente pour partie à une supervision de l'exécution des processus («Business Activity Monitoring »). Dans la réalité opérationnelle de traitement d'une crise civile, par exemple, l'ensemble de ces informations existent aujourd'hui sous la forme d'une trace écrite appelée «main courante». Une main courante sert à contrôler le déroulement de la réponse à la crise. Elle contient l'ensemble des événements, qu'ils soient juste sous forme d'un compte rendu de la bonne exécution d'un service ou bien des événements non programmés dont il faut jauger les causes et les effets.

Pour la cellule de gestion de crise, la main courante remplit les fonctions suivantes : (i) vérifier le bon déroulement des services, (ii) spécifier un compte rendu de la fin des services, (iii) noter les retards ou problèmes lors de l'exécution de services, tout en indiquant les raisons, (iv) noter tout nouveau risque ou conséquence qu'il faut traiter, (v) noter l'arrivée effective ou imminente de tout nouvel acteur, (vi) avoir la trace temporelle des phénomènes.

L'ensemble de ces informations est ensuite utilisé par la cellule de gestion de crise pour aider à la décision, à savoir : (i) anticiper à court terme l'évolution de la réponse de crise, notamment les problèmes possibles lors de la mise en exécution de la réponse, (ii) anticiper à moyen et long termes, i.e. essayer d'anticiper l'évolution de la crise ainsi que l'apparition de nouveaux risques aux conséquences non souhaitées.

La fiche de suivi a été structurée afin de permettre la synthèse de ces informations vitales. La figure 10 montre un exemple de fiche de suivi reprenant le cas d'études de la section 4. Au niveau de la réponse à la crise, le processus collaboratif a déjà commencé, et les pompiers viennent de commencer à secourir les personnes pendant que la croix rouge monte la tente de décontamination.

Comme l'illustre la figure 10, la fiche de suivi est composée de quatre tableaux. Les deux premiers portent sur les risques et leurs conséquences. Ils concernent la caractérisation de la crise. Les deux autres sont relatifs aux acteurs et aux services. Ils décrivent le système de traitement en cours de réalisation.

Les deux premiers tableaux ont un rôle identique. Ils résument l'ensemble des risques et conséquences de la crise ainsi que leur priorité de traitement (cf. section 4). Ils informent aussi sur la différence entre l'état réel et l'état supposé de chaque risque ou conséquence selon l'avancement de la réponse à la crise. L'état réel correspond à la réalité de la situation, il est renseigné par la cellule de crise. L'état supposé est déduit automatiquement en fonction de l'état des services, i.e. lorsque les services utilisés pour résoudre le risque A sont finis, l'état du risque A passe à résolu. 


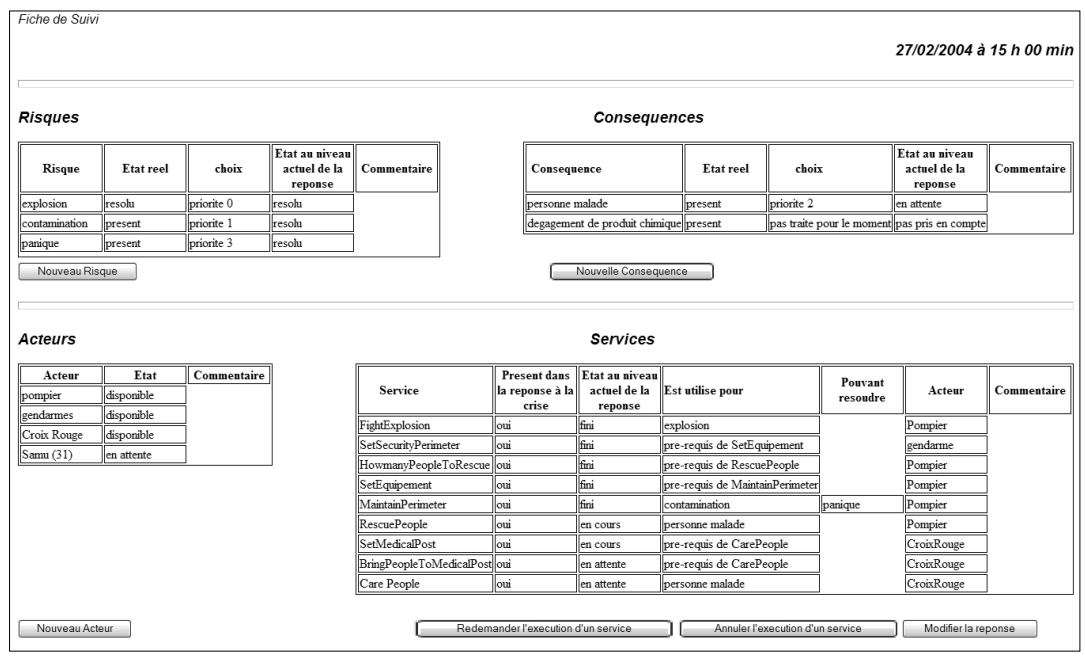

Figure 10. Exemple d'utilisation de la fiche de suivi

Quant aux deux autres tableaux (Acteurs et Services), ils renseignent sur le groupe d'acteurs sur l'évolution de déroulement du processus collaboratif en fonction de la qualité d'exécution des services (niveau de respect du contrat de services). Le tableau d'acteurs permet de détecter l'évolution structurelle de la composition du groupe d'acteurs de la cellule de gestion de crise. Le tableau de services quant à lui permet de détecter les variations de type fonctionnement. Il synthétise la bonne ou mauvaise exécution d'un service.

Quatre classes de diagnostic peuvent donc être dressées à partir d'une surveillance régulière de ces tableaux :

- si une nouvelle information entre dans un des deux premiers tableaux, la cellule de gestion de crise se retrouve face à une variation de caractéristiques de la crise, et doit donc modifier le modèle de crise, puis relancer la démarche de conception du système d'information collaboratif,

- si une différence entre les états d'une caractéristique de la crise (i.e. état réel et supposé) est notable, elle est significative d'une variation. Plusieurs solutions sont alors envisageables pour la cellule de gestion de crise, elle peut soit demander d'exécuter à nouveau un service, soit trouver de nouvelles compétences en interne ou externe,

- si une variation induit la redéfinition du processus collaboratif, la cellule de gestion de crise ne peut pas forcément choisir tout de suite les services à utiliser, une tâche abstraite sera alors définie qui sera remplacée au moment de son exécution par un service concret,

- si on modifie le champ de responsabilité des acteurs, alors le processus collaboratif doit être modifié. 


\subsection{Apporter de la flexibilité autour du processus collaboratif}

La flexibilité du système est étroitement liée à la faculté de reconcevoir et d'adapter l'exécution du processus collaboratif aux besoins de la cellule de gestion de crise. C'est une partie de l'étude qui s'inspire principalement de travaux sur la gestion de l'incertitude dans les systèmes de workflow. Nous citerons en particulier le workflow inter-organisationnel lâche (Andonoff $e t$ al, 2007) ou encore l'approche $\mathrm{ADEPT}_{\text {flex }}$ (Reichert et al, 1998) ou des propositions dans une veine apparentée (Van der Aalst, 1998). (Schonenberg et al., 2008) ont récemment proposé une synthèse de ces travaux avec une taxonomie des approches de la flexibilité dans les systèmes de workflow. Cette taxonomie, schématisée sur la figure 11, identifie quatre grandes classes d'approche :

- Flexibilité par conception (Flexibility by design): consiste à apporter de la flexibilité au niveau de la conception du processus en incluant des chemins d'exécution sur des activités alternatives. La sélection du chemin le plus approprié sera effectuée au moment de l'exécution.

- Flexibilité par déviation (Flexibility by deviation) : consiste à apporter de la flexibilité au moment de l'exécution en permettant de changer l'ordre d'exécution des activités sans les changer, i.e. permettre d'annuler, recommencer, sauter une tâche.

- Flexibilité par définition partielle (Flexibility by underspecification): consiste à définir partiellement le processus au niveau de la conception et de le compléter au moment de son exécution,

- Flexibilité par changement (Flexibility by change): consiste à modifier la définition du processus en cours d'exécution, par le bais d'insertion ou de suppression de tâches.

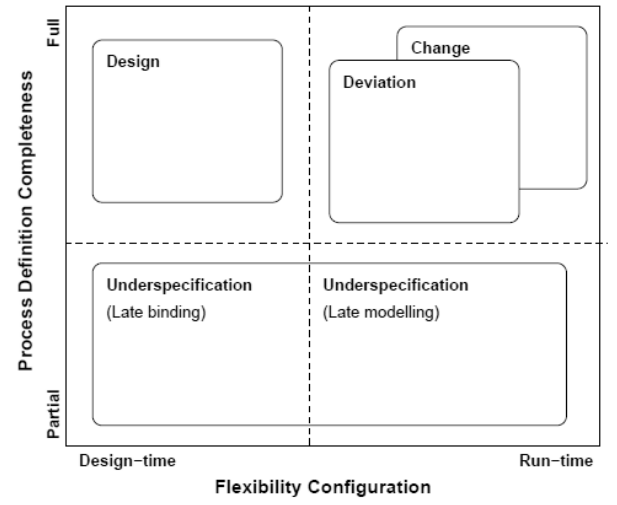

Figure 11. Différents types de flexibilité selon (Schonenberg et al, 2008) 
Une mise en relation des ces classes d'approche avec les types de variation de notre crise a guidé notre démarche. En effet, puisqu'une variation dans le fonctionnement peut impliquer l'arrêt, la relance d'une activité ou encore la modification du cours des événements lors de l'exécution, ce type de variation amène à développer une flexibilité de type déviation et/ou par changement. Dans le même ordre d'idée, lorsque la cellule de gestion ne peut pas décider au préalable quels services vont être exécutés pour traiter la crise, la situation est assimilable à une flexibilité de type définition partielle. Par contre, la flexibilité de conception ne trouve pas d'utilité dans notre cadre de travail. Car, par hypothèse, nous avons décidé de ne pas définir d'alternatives à un processus collaboratif caractérisant la volonté de la cellule de gestion de crise, qui se limite par conséquent à un seul scénario de traitement sur un temps donné (au sens où il n'y a pas de solution concurrente). Ce scénario est arrêté et un autre lui sera substitué en cas de variation. Cette position est légitimée par la nature trop imprévisible de l'évolution d'une crise. Une politique choisie peut être remise en cause pour plusieurs raisons (cf. section 1.3).

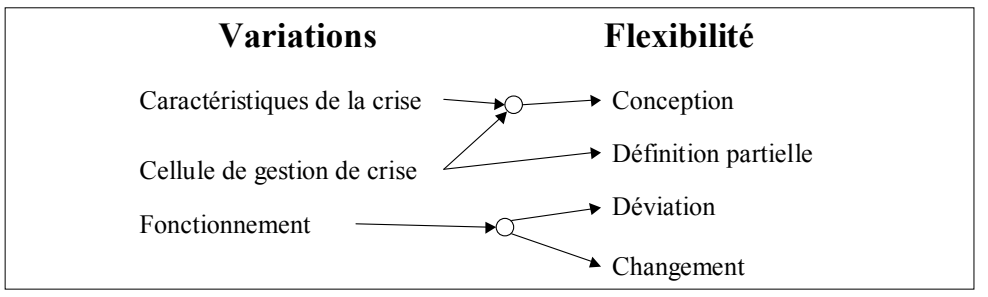

Figure 12. Relations entre variations et approches de flexibilité

La figure 12 résume notre démarche. En ce qui concerne les types de flexibilité par déviation et changement, des travaux sont en cours de réalisation par des partenaires du projet ISyCri, consistant en une évolution fonctionnelle du moteur d'orchestration de services de l'ESB PEtALS. Cette évolution doit permettre de suspendre, de reprendre ou d'arrêter l'exécution du processus collaboratif. D'autres travaux consistent en la réalisation d'un protocole de contractualisation (ContractNet) permettant la définition partielle du processus (Faure et al, 2009).

\section{Conclusions et travaux en cours}

Le système de gestion d'une crise s'assimile à un système de systèmes. L'utilisation d'un système d'information médiateur permet de coordonner les services métiers et les services applicatifs, et donc d'échanger les informations entre les différents systèmes d'information des acteurs. Pour être efficace, le système de systèmes doit évoluer concomitamment à la crise. La démarche de conception basée sur des techniques d'ingénierie dirigée par les modèles forme un support déterminant 
permettant de reconfigurer un médiateur pour coller aux exigences de la cellule faisant suite à chaque variation détectée du besoin dans l'espace de collaboration.

Comme le système de systèmes de réponse à la crise a besoin d'être agile, la notion d'agilité est caractérisée par les capacités de réactivité et de flexibilité. Si la réactivité est atteinte grâce à l'adaptation ponctuelle des services des contributeurs qui sont facilement accessibles, la flexibilité se décompose en deux approches issues des travaux en workflow : la flexibilité par conception. L'implémentation de ces techniques est possible grâce à des services de transformation de modèles embraqués dans la solution qui permettent en quelque sorte une autoconfiguration du médiateur. Le niveau de reprise de la chaîne de conception est décidé grâce à l'utilisation d'une fiche de suivi qui permet de comparer la situation réelle à la situation théorique déduite de l'avancement du processus.

Le travail de test des solutions exposées dans cet article est en cours de réalisation. Ces tests devraient pouvoir faire la preuve de l'adéquation d'ensemble de notre approche à la problématique soulevée. Un autre travail en cours essaye de développer une approche permettant d'analyser les raisons de changement d'une réponse afin d'améliorer la phase de préparation à la gestion de crise et par conséquent la déduction du processus collaboratif. Ce travail se base sur l'idée de coupler les avantages de flexibilité du processus à l'analyse des changements présentée dans (Weber et al, 2005). Dans cet article, les auteurs proposent de combiner les avantages de l'approche ADEPTflex (Reichert et al, 1998) à l'approche CBRFlow (Weber et al.; 2004) afin de pouvoir profiter au maximum du retour d'expérience.

\section{Bibliographie}

Andonoff E., Bouaziz W., Hanachi C., "Protocol Management Systems as a Middleware for Inter-Organizational Workflow Coordination", IJCSA 4, 2007, p. 23-41.

Andonoff E., Chapurlat V., Hanachi C., Montmain J., Sibertin-Blanc C., Tahir O., Etude des modes de pilotage dynamique du Processus Collaboratif, livrable du projet ISyCri, 2008.

Altlay N. and Green W., "OR/MS research in disaster operations management”, European Journal of Operational Research, v175 i1, p. 475-493.

Aubert B., Dussart A., Systèmes d'information Inter-Organisationnels, Rapport Bourgogne, CIRANO, mars 2002.

Badot O., Théorie de «l'entreprise agile », L'Harmattan, 1997.

Beamon B.M. and Kotleba S.A., "Inventory modelling for complex emergencies in humanitarian relief operations", International Journal of Logistics: Research and Applications, v9 i1, p. 1-18.

Bénaben F., Pignon J.P., Hanachi C., Lorré J.P., Chapurlat V., «Interopérabilité des systèmes en situation de crise ", WISG'07, ANR \& DGA Interdisciplinary Workshop on Global Security, Troyes, 2007. 
Bénaben F., Touzi J., Rajsiri V., Lorré J.P., Pingaud H., «L’Interopérabilité des systèmes d'information comme moyen vers l'intégration de l'écosystème industriel », $7^{e}$ Congrès international de génie industriel, Trois-Rivières, Québec, 2007.

Bézivin J., «Sur les principes de base de l'ingénierie des modèles », L'objet, Où en sont les objets ?, vol. 10, n 4, 2004

Bézivin J., Dupé G., Jouault F., Pitette G., Rougui J.E., "First Experiments with the ATL Model Transformation Language: Transforming XSLT into Xquery", OOPSLA'03, Anaheim, 2003.

Bouzguenda L., Coordination multi-agents pour le Workflow inter-organisationnel lâche, Thèse de doctorat, IRIT, 2006.

Devlin E., Crisis Management Planning And Execution, p. 528, 2006.

Faure C., Andonoff E., Hanachi C., Sibertin-Blanc C., Salatge N., «Flexibilité de processus de gestion de crise par intégration de protocoles d'interaction », INFORSID'09, Toulouse, 2009.

Hansen-Glize C., « de la maîtrise des crises », Techniques de l’ingénieur, 2008.

Jouault F., Contribution à l'étude des langages de transformation de modèles, Thèse de doctorat, Université de Nantes, 2006.

Kidd T.P., Agile Manufacturing: Forging New Frontiers, London, Addison-Wesley, 1994.

Lindberg P., "Strategic manufacturing management: a proactive approach", International Journal of Operation and Production Management, vol 10, $\mathrm{n}^{\circ}$ 2, 1990, p. 94-106.

Lagadec P., « La gestion des crises », Ediscience international, p. 326, 1992.

Luzeaux D., Ruault JR., Système de systèmes, concepts et illustrations pratiques, Paris, Lavoisier, 2008.

Luzeaux D, Ruault JR., Système de systèmes, méthodes et outils, Paris, Lavoisier, 2008.

OMG, MDA guide, 2006, http://www.omg.org/docs/omg/03-06-01.pdf

Maier M.W., « Architecting principles for systems-of-systems », Systems Engineering, vol. 1, $\mathrm{n}^{\circ} 4,1998$, p. $267-284$.

Pingaud H., «Rationalité du développement de l'interopérabilité dans les organisation », Management des technologies organisationnelles - Journées d'étude 2009, p. 19-30 2009.

Rajsiri V., Knowledge-based system for collaborative process specification, Thèse de doctorat, Institut National Polytechnique Toulouse, 2009.

Reichert M., Dadam P., "ADEPTflex - supporting dynamic changes of workflows without losing control”, Journal of Intelligent Information Systems 10, 1998, p. 93-129.

Ryfman P., «La question humanitaire - histoire, problématiques, acteurs et enjeux de l'aide humanitaire internationale », Ellipses Marketing, 1999, p. 208.

Rueppel U., Wagenknecht, “A.: Improving Emergency Management by Formal Dynamic Process-Modelling", Proceedings of the $24^{\text {nd }}$ Conference on Information Technology in Construction (W78), Maribor, Slovenia, July 2007, p. 559-564. 
Roadmap EI, http://cordis.europa.eu/ist/ict-ent-net/ei-roadmap_en.htm, 2008

Schonenberg H., Mans R., Russell N., Mulyar M., Van der Aalst W.M.P., "Process Flexibility: A Survey of Contemporary Approaches (Extended Version)", J.L.G. Dietz, A. Albani, J. Barjis (Eds.), Advances in Enterprise Engineering (4 ${ }^{\text {th }}$ International Workshop CIAO! and $4^{\text {th }}$ International Workshop EOMAS, held at CAiSE 2008, Montpellier, 2008, Proceedings). Lecture Notes in Business Information Systems, vol. 10, p. 16-30). Berlin: Springer.

Sharifi H., Zhang Z., "A methodology for achieving agility in manufacturing organizations: An introduction", International Journal of Production Economics, vol. 62, 1999, p. 7-22.

Tomasini R. et Van Wassenhove Luk N., Genetically Modified (GM) Food Donations and the Cost of Neutrality: Logistics Response to the 2002 Food Crisis in Southern Africa. INSEAD case no. 03/2004-5169, 2004.

Touzi J., Bénaben F., Pingaud H., "Model Transformation of Collaborative Business Process into Mediation Information System”, IFAC'08, Elsevier, Seoul, 2008.

Touzi J., Aide à la conception de Système d'Information Collaboratif support de l'interopérabilité des entreprises. Thèse de doctorat, Institut National Polytechnique Toulouse, 2007.

Truptil S., Bénaben F., Couget P., Lauras M., Chapurlat V., Pingaud H., "Interoperability of Information Systems in Crisis Management: Crisis Modeling and Metamodeling", INTEROP-ESA'08, Berlin, 2008.

Truptil S., Bénaben F., Pingaud H., "Collaborative process design for Mediation Information System Engineering”, ISCRAM'09, Göteborg, 2009.

Van der Aalst W.M.P., Basten T., "Inheritance of Workflows: An approach to tackling problems related to change", Computing Science Reports 99/06, Eindhoven University of Technology, Eindhoven, 1999.

Van der Aalst W.M.P., "Changing Workflows: On the interplay between flexibility and support”, J. Desel, A. Oberweis, W. Reisig, and G. Rozenberg, editors, Petri Nets and Business Process Management,. Dagstuhl Seminar Report, 217, Dagstuhl, July 1998, p. 5-6.

Van Wassenhove Luk N., "Humanitarian aid logistics: supply chain management in high gear", Journal of the Operational Research Society, 2006, p. 475-489.

Vernadat F., Enterprise Modelling and Integration, London, Chapman \& Hall, 1996.

Vernadat F., "Interoperable enterprise systems: architecture and methods", Plenary lecture, IFAC/INCOM, Saint-Etienne, 2006.

Weber B., Wild W., Breu R., "CBRFlow : Enabling adaptive workflow management through conversional case-based reasoning”, ECCBR'04, Madrid, 2004.

Wiederhold G., "Mediators in the Architecture of Future Information Systems", IEEE Computer magazine, vol 25, $\mathrm{n}^{\circ} 3,1992$, p. 38-49. 